\title{
Caracterización del co-encapsulamiento de Lactobacillus plantarum y ácidos grasos omega-3 en una matriz de alginato-pectina
}

\author{
Co-encapsulation and characterization of Lactobacillus plantarum and omega-3 fatty acids \\ in alginate-low metoxil pectin matrix
}

\begin{abstract}
Silvia Gabriela López-Fernández², Silvia Guadalupe Fernández Michel², Rosa Idalia Armenta Corral', Alfonso GarcíaGalaz', Silvia Carolina Moreno-Rivas ${ }^{1}$, Luz Vázquez-Moreno L' ${ }^{1}$, Gabriela Ramos-Clamont Montfort ${ }^{1 *}$

1 Laboratorio de Bioquímica de Proteínas y Glicanos, Coordinación de Ciencia de los Alimentos, Centro de Investigación en Alimentación y Desarrollo A.C. Carretera Gustavo Enrique Astiazarán No 46, Col, la Victoria. Hermosillo Sonora México CP83304

2 Facultad de Ciencias Biológicas. Universidad Autónoma de Coahuila. Carretera Torreón - Matamoros Km. 7.5. Torreón, Coahuila, México. C.P. 27104.
\end{abstract}

\section{RESUMEN}

Los alimentos funcionales producen un efecto benéfico en la salud de quien los consume. Algunos de ellos se desarrollan adicionando probióticos o compuestos bioactivos, que son sensibles a condiciones adversas presentes durante la vida de anaquel del alimento o al pasar por el tracto gastrointestinal. En ambos casos, una alternativa para protegerlos, es encapsularlos en matrices grado alimenticio. Se co-encapsularon Lactobacillus plantarum y ácidos grasos omega-3 $(\Omega 3)$ en una matriz de alginato-pectina. Los $\Omega 3$ se obtuvieron por extracción en frío de aceite de linaza; su análisis cuantitativo por cromatografía de gases mostro un contenido del $58 \%$. La relación de matriz polimérica que produjo mejores cápsulas fue 2:1, usando concentraciones de $1.5 \%$ y $2.0 \%$, de alginato y pectina, respectivamente. La mejor relación matriz-probiótico-linaza fue de 5.0:1.0:1.0 (suspensión celular conteniendo aproximadamente $2.8 \mathrm{x}$ $10^{8} \mathrm{UFC} / \mathrm{mL}$ ). Se obtuvieron cápsulas esféricas con carga superficial, negativa $(-4.6 \pm 0.41 \mathrm{mV})$ y diámetros entre 150 y $200 \mu \mathrm{m}$. La eficiencia de encapsulamiento de L. plantarum fue del $85 \%$, observándose una disminución de $0.7 \pm 0.14$ Log UFC/g, después de liofilizar las microcápsulas para mejorar su estabilidad durante el almacenamiento. Después de 30 días a $-20{ }^{\circ} \mathrm{C}$, no se observaron diferencias $(p>0.05)$ en la sobrevivencia del probiótico, ni cambios en la concentración de peróxidos del aceite co-encapsulados, indicando que el encapsulamiento protegió tanto a L. plantarum como a los $\Omega 3$. Estos micro-encapsulados podrían utilizarse en la formulación de productos funcionales debido a su bajo costo de producción y fácil estabilización.

Palabras clave: Micro-encapsulados funcionales, probióticos, bioactivos, aceite de linaza, alginato-pectina.

\section{ABSTRACT}

Functional foods are those with a potentially positive effect on consumer health. Some of them are developed by adding probiotics or bioactive compounds, which are sensitive to adverse conditions present during food shelf life as well as during the passage through the gastrointestinal tract. In both cases, an alternative to provide protection is the encapsulation in food grade matrices. Lactobacillus plantarum and omega-3 fatty acids $(\Omega 3)$ were co-encapsulated in an alginate-pectin matrix. The $\Omega 3$ were obtained by cold extraction of linseed oil, quantitative analysis conducted by gas chromatography showed $\Omega 3$ content of $58 \%$. The best capsules were obtained with a polymer matrix ratio of 2 : 1 , using concentrations of $1.5 \%$ and $2.0 \%$, of alginate and pectin, respectively. The best probiotic-linseed oil ratio was 5.0: 1.0: 1.0 (cellular suspension of approximately $2.8 \times 10^{8}$ $\mathrm{CFU} / \mathrm{mL}$ ). The morphological study showed spherical microcapsules with a negative surface charge $(-4.6 \pm 0.41 \mathrm{mV})$ and diameters between 150 and $200 \mu \mathrm{m}$. Encapsulation efficiency of $L$. plantarum was $85 \%$, with a decrease of $0.7 \pm 0.14 \mathrm{Log}$ $\mathrm{CFU} / \mathrm{g}$, after lyophilizing the microcapsules to improve their stability during storage. After 30 days at $-20^{\circ} \mathrm{C}$, no differences were observed $(p>0.05)$ in the survival of the probiotic, nor changes in the concentration of co-encapsulated linseed oil peroxides These results indicating that the encapsulation protected both probiotic and $\Omega 3$. These co-microcapsules could have utility in functional food formulations due to simple and cost effective production and stabilization.

Keywords: Functional microencapsulates, probiotics, bioactives, linseed oil, alginate-pectin.

\section{INTRODUCCION}

La tendencia hacia el consumo de alimentos más saludables ha despertado el interés de la población por los alimentos funcionales. Algunos de estos alimentos contienen o se les han adicionado, compuestos bioactivos que producen algún beneficio específico para la salud. Ejemplos de bioactivos son fitoesteroles, péptidos antihipertensivos, carotenos, antioxidantes y ácidos grasos poliinsaturados como los $\Omega 3$ (Sampson, 2015). Los probióticos son "microorganismos benéficos que producen un beneficio a la salud cuando se consumen vivos en cantidad suficiente" (FAO/OMS, 2002), también se cuentan entre los bioactivos que se añaden a los alimentos (Ghidurus et al., 2015). En particular, el pro-

*Autores para correspondencia: Gabriela Ramos-Clamont Montfort Correo electrónico: gramos@ciad.mx

Recibido: 22 de abril de 2018 
biótico Lactobacillus plantarum ha demostrado proteger al intestino de la disrupción provocada por enteropatógenos (Wu et al., 2016), disminuir los niveles de triglicéridos y en general, el síndrome metabólico en modelos murinos obesos (Stevenson et al., 2015; Ben Salah et al. 2013). También se le han atribuido propiedades antioxidantes, antimicrobianas, antiinflamatorias y anti cancerígenas y ha sido estudiado como tratamiento complementario en enfermedades como: Parkinson, Alzheimer, diabetes mellitus, síndrome del colon irritable (llavenil et al., 2015; Barreto et al., 2014; Woo et al., 2014; Jeun et al., 2010).Otros compuestos bioactivos son los ácidos grasos $\Omega 3$, que por lo general se consumen poco en la dieta occidental. Diversos estudios epidemiológicos y ensayos clínicos aleatorizados, muestran un efecto positivo entre el consumo de ácidos grasos $\Omega 3$ y la reducción de obesidad, riesgo cardiovascular, asma, enfermedades inflamatorias como la artritis y la mejora del desarrollo cognitivo y neurológico (Baker et al., 2016; Chowdhury et al., 2014; Barros et al., 2011). Las principales fuentes de ácidos $\Omega 3$ son de origen marino, como el aceite que se encuentra en salmón, sardina y atún. Además, existe un interés creciente en las propiedades y consumo de fuentes vegetales de $\Omega 3$, como el aceite de linaza que contiene hasta $50 \%$ de ácido alfa linolénico (ALA; 18:3w-3) (Baker et al., 2016).

La adición de compuestos bioactivos a los alimentos implica desafíos tecnológicos que la industria alimentaria tiene que vencer. En el caso de los $\Omega 3$, destaca su susceptibilidad a reacciones de rancidez oxidativa, que derivan en la pérdida de su bioactividad y dan lugar a la formación de peróxidos y de moléculas que producen colores y sabores desagradables en el alimento y la pérdida de su valor nutricional (Comunian y Favaro-Trindade, 2016). Otra desventaja de los $\Omega 3$ es su escasa miscibilidad en el agua, lo que dificulta su adición a bebidas y a una gran cantidad de alimentos (Baker et al., 2016). Por su parte, los probióticos, cuya condición para ejercer un efecto benéfico es llegar vivos y establecerse en el intestino en cantidad suficiente (106-10 $\mathrm{UFC} / \mathrm{g}$ de alimento), tienen que enfrentar una serie de estreses ambientales que derivan en la pérdida de su viabilidad y a los que están expuestos, desde la producción del alimento, hasta su consumo; entre estos estreses destacan la exposición a oxígeno, temperatura y fuerza iónica adversas, bajos $\mathrm{pH}$ gástricos, etc. (Morales y Ruiz, 2016).

El encapsulamiento es una alternativa tecnológica para la protección de compuestos bioactivos que se adicionan a los alimentos. Básicamente, consiste en crear una barrera entre el bioactivo y el alimento, que en el caso de los $\Omega 3$ protege de la oxidación y facilita su incorporación a alimentos acuosos; en el caso de los probióticos, los resguarda del estrés ambiental que disminuyen su viabilidad (Comunian y Favaro-Trindade, 2016; Morales y Ruiz, 2016). Diversos estudios demuestran la efectividad del microencapsulamiento para preservar la estabilidad de los $\Omega 3$ y la viabilidad de los probióticos en general y de los ALA; $18: 3 \omega-3$ y L. plantarum en particular (Avramenko et al., 2016; Li et al., 2016; Rajam y Anandharamakrishnan, 2015; Carneiro et al.,
2013). Sin embargo, existen pocos trabajos donde ambos bioactivos se encapsulan para aprovechar sus beneficios, al ser añadidos a un mismo alimento. Eratte et al. (2016b, 2015), encapsularon L. casei y aceite de atún en una matriz formando coacervados de proteínas de suero lácteo y goma arábiga. Encontraron que la viabilidad del microorganismo encapsulado se preserva de manera más eficiente en presencia del aceite (aproximadamente ciclo logarítmico mayor que los controles sin encapsular). Es importante investigar si la presencia de $\Omega 3$ promueve el crecimiento de diferentes probióticos, añadiendo a los estudios controles en los que se encapsule microorganismos en ausencia del aceite.

El alginato es un polisacárido que se forma por regiones homopoliméricas de ácido gulurónico (GGGGG) y ácido manurónico (MMMMM); también existen, regiones heteropolímericas donde se combinan, M y G (Guo et al., 2016). Este polisacárido se encuentra entre las matrices más utilizadas para microencapsular bioactivos que se añadan a los alimentos. Los alginatos son comestibles e inocuos (GRAS, por sus siglas en inglés); gelifican fácilmente en presencia de calcio $\left(\mathrm{Ca}^{+2}\right)$, formando hidrogeles que se ajustan al modelo "tipo caja de huevo" (Morales y Ruiz, 2016). En este modelo, la interacción se establece entre los grupos carboxilo de las regiones GGGG y el $\mathrm{Ca}^{+2}\left(\mathrm{COO}^{-}{ }^{+} \mathrm{Ca}^{+} \mathrm{COO}\right)$. Para mejorar su capacidad protectora se ha propuesto mezclarlo con otros polisacáridos. Una alternativa es mezclarlos con pectinas de bajo metoxilo que también forman geles en presencia de $\mathrm{Ca}^{+2}$. En este caso, las interacciones $\mathrm{COO}^{+}{ }^{+} \mathrm{Ca}^{+} \mathrm{COO}$ ), se establecen entre los grupos carboxilo del ácido galacturónico que no se encuentran metoxilados (Guo et al., 2016).Por todo lo anterior, el objetivo de este trabajo fue caracterizar el co-encapsulamiento de $L$. plantarum y ácidos grasos $\Omega 3$ en una matriz de alginato-pectina, determinando la viabilidad del probiótico, y la estabilidad de los ácidos grasos después de liofilizar las cápsulas y de 30 días de almacenamiento a $-20^{\circ} \mathrm{C}$.

\section{MATERIALES Y MÉTODOS Materiales}

Los medios de cultivo se obtuvieron de Difco (Difco Laboratories/Becton, Dickinson and Co, MD, USA). El alginato de sodio, las pectinas de bajo metoxilo y resto de los reactivos se adquirieron de Sigma-Aldrich (St. Louis, MI, EUA).

\section{Extracción del aceite de linaza y determinación de su perfil de ácidos grasos}

Las semillas de linaza (Linum usitatissimum), se adquirieron en 2 tiendas de productos naturales de Torreón Coahuila; se utilizó la variedad canadiense de semillas marrón, obteniéndose 3 muestras de $2 \mathrm{~kg}$ en cada tienda. La semilla se trituró durante $3 \mathrm{seg}$ en un molino WSG60 (Waring Co, E.U.A). Después de tamizar (tamiz \# 4), el producto de la molienda se introdujo en una prensa de tornillo sin fin DLZYJ02 (Dulong Co, EUA) extrayendo el aceite por prensado en frío, cuidando que la temperatura durante todo el proceso 
fuera menor a $40^{\circ} \mathrm{C}$. El aceite obtenido se centrifugó (Hermle Z. 326K, E.U.A) a $105478 \mathrm{xg}$ a $4^{\circ} \mathrm{C}$ durante $30 \mathrm{~min}$ y se colocó en recipientes de vidrio ámbar a $4^{\circ} \mathrm{C}$, hasta su uso.

El perfil de ácidos grasos del aceite se determinó por cromatografía de gases, como ésteres de metilo, preparando las muestras según la metodología descrita por Slover y Lanza (1979). Las muestras se inyectaron en un cromatógrafo de gases (Agilent Technologies 6820, EUA), con detector de ionización de flama; se usó una columna capilar CP-Sil 88 (Agilent, EUA), fase estacionaria fase estacionaria interna de Ciano propil silicón $100 \%$, grosor de $0.20 \mu \mathrm{m}$. Las condiciones de $120^{\circ} \mathrm{C}$ por 5 min; se llegó a $210^{\circ} \mathrm{C}$ a razón de $3{ }^{\circ} \mathrm{C} / \mathrm{min}$; se incrementó la temperatura hasta $240^{\circ} \mathrm{C}$ a $1^{\circ} \mathrm{C} /$ min hasta que se completó un tiempo de corrida de 72 y 67 min para el $\Omega 3$ y 6 respectivamente. Las temperaturas del inyector y del detector fueron de $260^{\circ} \mathrm{C}$. Se utilizó helio grado 4.7 como acarreador. Los ácidos grasos se identificaron por comparación con los tiempos de retención de los estándares de referencia.

\section{Bacteria y condiciones de cultivo.}

La cepa de $L$ plantarum se adquirió de la American Type Culture Colection (ATCC 14917). La bacteria liofilizada se inoculó en $5 \mathrm{~mL}$ de caldo MRS (Man, Rogosa y Sharpe), que contenía $0.5 \%$ de L-cisteína. Se incubó en aerobiosis a $37{ }^{\circ} \mathrm{C}$ durante $48 \mathrm{~h}$. Después de una nueva resiembra a las mismas condiciones, el cultivo obtenido se almacenó a -40 ${ }^{\circ} \mathrm{C}$ en viales de crio-cultivo de $1.5 \mathrm{~mL}$, y se adicionó $20 \%$ de glicerol estéril como crioprotector.

Para obtener la biomasa a encapsular, la bacteria se inoculó en caldo MRS con $0.05 \%$ de L-cisteína y se incubó a 37 ${ }^{\circ} \mathrm{C}$, durante $24 \mathrm{~h}$. Las células se recuperaron por centrifugación a $1370 \mathrm{xg}$ durante $15 \mathrm{~min}$ a $15^{\circ} \mathrm{C}$, en una centrifuga Allegra 6R (Beckman. EUA). La biomasa precipitada se lavó 3 veces con solución salina estéril al $0.85 \%$. Posteriormente, se ajustó con esta misma solución a una absorbancia de 0.8 a $620 \mathrm{~nm}$,

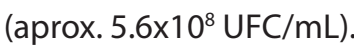

\section{Crecimiento de L. plantarum en presencia de aceite de linaza}

Para determinar el efecto del aceite de linaza sobre el crecimiento de L. plantarum se hizo un microensayo siguiendo la metodología propuesta por Hajlaoui et al. (2016), con modificaciones. El volumen total de cada pozo fue de 200 $\mu \mathrm{L}$, utilizando $140 \mu \mathrm{L}$ de caldo MRS con $0.5 \%$ de cisteína (MRS-cys) $50 \mu \mathrm{L}$ de aceite diluido en solución salina a la concentración correspondiente y $10 \mu \mathrm{L}$ de inóculo conteniendo $2.8 \times 10^{8} \mathrm{UFC} / \mathrm{mL}$ de L. plantarum. El aceite de linaza se diluyó en solución salina añadiéndole $10 \%$ de dimetil-sulfóxido; se obtuvieron concentraciones de aceite por doble dilución seriada, desde $8 \%$ hasta $0.125 \%$. Las cajas se prepararon bajo flujo laminar, se taparon y colocaron en un lector de Elisa Anthos Zenyth 340st (Alcobendas, Madrid), incubándolas a $37^{\circ} \mathrm{C}$ durante $48 \mathrm{~h}$. El aparato se programó para realizar mediciones de absorbancia a $620 \mathrm{~nm}$, cada 30 min introduciendo un ciclo de agitación a intensidad media cada 20 seg. De cada pozo se generó una curva de crecimiento.Se hicieron dos experimentos independientes con un total de 16 curvas de crecimiento por concentración de aceite analizada. Para obtener los parámetros de crecimiento se utilizó el programa DMfit versión 2.0 (Norwich, United Kingdom) (Baranyi y Roberts, 1994). Como controles se utilizaron: caldo MRS-cys sin inocular, caldo MRS-cys inoculado y caldo MRC-cys sin inocular con aceite, sin inocular.

\section{Microencapsulamiento}

Primero se probaron diferentes combinaciones de a) alginato-pectina (matriz) y b) matriz-probiótico-aceite, para obtener cápsulas consistentes. Estas cápsulas se hicieron poniendo la mezcla en una jeringa para insulina y goteándola a un baño con agitación conteniendo $\mathrm{CaCl}_{2} 0.3 \mathrm{M}$, con 0.1 $\%$ de tween 20. Primero se estandarizaron las condiciones para la matriz. Se hicieron cápsulas de pectina al $2 \%$ (T1); de alginato (1\%)-pectina (2\%) relación 2:1 (T2) y de alginato (1.5\%)-pectina (2\% ), relación 2:1 (T3). Con la mejor combinación se hicieron cápsulas matriz-probiótico- $\Omega 3$ usando las proporciones, 5.0:2.5:2.5, 5.0:1.0: 2.5 y 5.0: 1.0:1.0. Finalmente, se trabajó con una proporción matriz-probiótico-omega-3, 5.0:1.0:1.0, en la que la matriz estuvo constituida por proporción 2:1 de solución de alginato al $1.5 \%$ y solución de pectina al $2 \%$. Para miniaturizar las cápsulas se utilizó un equipo Encapsulator B-395 Pro (Buchi labortechnik, AG) con una boquilla de $200 \mu \mathrm{m}$, frecuencia de $2000 \mathrm{~Hz}$., voltaje de 1500 volts, agitación de $90 \%$, presión de 650 mbar y una velocidad de alimentación de $0.6 \mathrm{~mL} / \mathrm{min}$. La suspensión alginato-pectina conteniendo al probiótico se aplicó al equipo a través de mangueras de ascensión, mientras que el aceite se aplicó con una jeringa automática, siendo co-extruidos simultáneamente hacia una solución de $\mathrm{CaCl}_{2} 0.3 \mathrm{M}, 0.1 \%$ tween 20, a través de una boquilla concéntrica, para asegurar que el aceite quedara en el centro de la microcápsula. Los cálculos se realizaron para que el contenido final de L. plantarum encapsulado fuera aproximadamente de $2.8 \times 10^{8} \mathrm{UFC} /$ $\mathrm{mL}$ (Log $8.4 \mathrm{UFC} / \mathrm{mL}$ ) y el de aceite $8 \%$.

Los microencapsulados obtenidos se dejaron en agitación a 2 xg por $30 \mathrm{~min}$, dentro del recipiente de reacción del equipo y durante $90 \mathrm{~min}$, en reposo en refrigeración a 4 ${ }^{\circ} \mathrm{C}$, para continuar con el entrecruzamiento. Posteriormente, se lavaron 3 veces con agua bidestilada para eliminar el $\mathrm{Ca}^{+2}$ que no reaccionó. Enseguida de este tratamiento, se dividieron en dos grupos. Con el primero se determinó la eficiencia de encapsulamiento y la caracterización de las cápsulas, mientras que las cápsulas del segundo grupo se liofilizaron (Virtis Benchtop 6.6 SP Industries, Gardiner, NY, EUA. $-54{ }^{\circ} \mathrm{C}$ por $48 \mathrm{~h}$ ) para los estudios de viabilidad. Se prepararon 5 lotes diferentes de microencapsulados.

\section{Caracterización de las microcápsulas Tamaño}

Las cápsulas se dispersaron en agua deionizada (10\% $\mathrm{p} / \mathrm{v})$, determinándose el tamaño por difracción laser en un equipo Mastersizer, WR14 1XZ, (Malvern, UK.) Los tamaños 
se documentaron usando el software Mastersizer Micro Ver. 2.19 (Malvern, UK). El análisis se realizó por triplicado.

\section{Carga superficial}

La carga superficial de las microcápsulas se determinó en un analizador de potencial zeta (Zetasizer Nano-ZS90, Malvern, UK) a $25^{\circ} \mathrm{C}$. Las microcápsulas se dispersaron (10 $\%, \mathrm{p} / \mathrm{V})$ en agua deionizada. Se hicieron 3 corridas de 100 determinaciones cada una, usando el software Zetasizer 7.01 (Malvern, UK).

\section{Morfología}

La morfología de las microcápsulas se determinó por estereoscopía (Stem DV4, Zeiss, Alemania), y por microscopía óptica (Quodmaster 100 modelo M1000-D, Swift, EUA). Los estudios se hicieron por triplicado.

\section{Eficiencia de Encapsulamiento}

Para estimar la eficiencia de encapsulamiento, se calculó la cantidad de bacterias que se liberaron de las cápsulas siguiendo la técnica descrita por Mokarram et al. (2009). Las microcápsulas $(1 \mathrm{~g})$ se llevaron a un volumen de $99 \mathrm{~mL}$ en citrato de sodio estéril $1 \%(\mathrm{w} / \mathrm{v}), \mathrm{pH} 6.0$ y se agitaron suavemente durante $10 \mathrm{~min}, \mathrm{a} 25^{\circ} \mathrm{C}$. La sobrevivencia de la bacteria liberada se determinó tomando muestras de la dispersión y haciendo diluciones seriadas en agar MRS-cys. Posteriormente, se incubaron por $48 \mathrm{~h}$ a $37^{\circ} \mathrm{C}$. Se registró el crecimiento (Log UFC/mL) y se reportó como la media de 2 repeticiones con 2 observaciones para cada dilución. El rendimiento de encapsulamiento (RE), que es una medida de eficacia del atrapamiento y la supervivencia de células viables durante la encapsulación, se calculó de la siguiente manera:

$\mathrm{RE}=(\mathrm{N} / \mathrm{N} 0) 100$

Donde:

$\mathrm{N}$ = número de células viables liberadas de las microcápsulas

NO = número de células añadidas a la mezcla de polímeros.

Como controles se usaron microencapsulados de $L$. plantarum sin la presencia de aceite

Efecto de la liofilización y del almacenamiento en congelación en la viabilidad del $L$. plantarum microencapsulado

Las cápsulas se liofilizaron y se determinó la viabilidad de L. plantarum, al término de este proceso y después de 30 días de almacenamiento a $-20^{\circ} \mathrm{C}$. Se utilizó la técnica de dilución en placa en agar MRS-cys, incubando a $37^{\circ} \mathrm{C}$ durante 48 h. Para asegurar la liberación del microorganismo, se usó la misma técnica con citrato de sodio, descrita en la sección de eficiencia de encapsulamiento. El crecimiento se registró en (log UFC/mL) y se reportó como la media de tres repeticiones con tres observaciones para cada dilución. Como controles se usaron microencapsulados de L. plantarum sin la presencia de aceite.

\section{Estabilidad del aceite encapsulado}

La estabilidad del aceite encapsulado se determinó mediante el índice de acidez y el índice de peróxidos de acuerdo a la Norma Mexicana NMX-F-101-1987 y a la Norma Mexicana NMX-F-154-1987, respectivamente. Ambos análisis se realizaron por triplicado con dos observaciones por réplica, determinando la estabilidad a los 0, 5,10 y 20 días de almacenamiento $\mathrm{a}-40^{\circ} \mathrm{C}$.

\section{Análisis estadístico}

Se hizo un análisis de varianza para el efecto de los $\Omega 3$ en el crecimiento de $L$. plantarum, viabilidad y estabilidad de aceite. Todos los datos se analizaron en el paquete estadístico NCSS (Versión 2007). Cuando existió diferencia significativa, se realizó una comparación de medias por la prueba de TukeyKramer. Las significancias estadísticas fueron consideradas a un nivel de probabilidad en el error de 0.05 . Al resto de los análisis se les aplicó la estadística descriptiva reportando las medias y desviaciones estándar.

\section{RESULTADOS Y DISCUSIÓN}

\section{Perfil de ácidos grasos del aceite de linaza-}

La Figura 1 muestra el cromatograma típico de la composición de los ácidos grasos presentes en el aceite de linaza. Se obtuvo una concentración de $58.0 \%$ de ácidos $\Omega 3$ y 14.7 $\%$ de ácidos $\Omega 6$, con tiempos de retención de 71.6 min y 67.2 $\mathrm{min}$, respectivamente. Otros ácidos grasos presentes fueron el palmítico (5.4\%), el esteárico (3.0\%) y el oleico (19.0\%). Estos resultados son similares a los encontrados por Jiménez et al. (2013), para otras variedades de linaza.

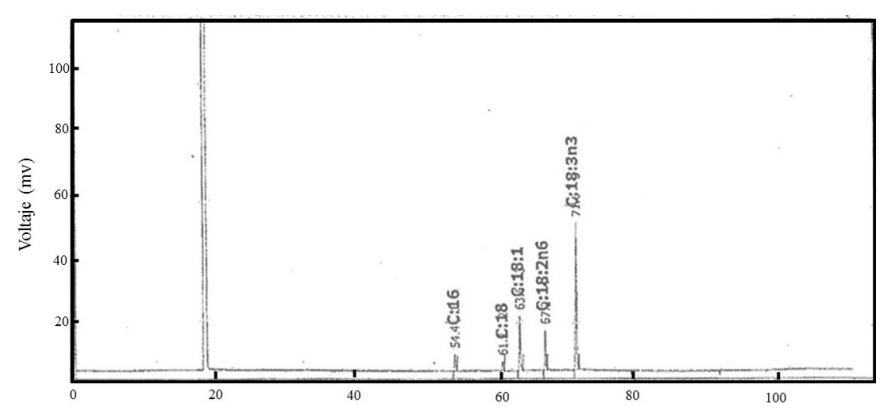

Figura 1. Perfil de ácidos grasos del aceite de linaza. Figure 1. Fatty acid profile of flaxseed oil.

\section{Crecimiento de $L$. plantarum en presencia de aceite de linaza}

Para determinar el efecto de los ácidos $\Omega 3$ sobre el desarrollo de $L$. plantarum se hicieron cinéticas de crecimiento en presencia de diferentes concentraciones de aceite de linaza, utilizando la técnica de micro-dilución en placa. La Tabla 1 muestra los parámetros de crecimiento, encontrándose diferencias $(p<0.05)$ entre la duración de la fase de acondicionamiento de los controles y tratamientos. En los controles sin aceite, la fase de acondicionamiento tuvo una duración de $2.33 \pm 0.12 \mathrm{~h}$, que disminuyó a un promedio de $1.83 \pm 0.18$ h, $(21 \%)$, cuando la concentración de aceite 
Tabla1. Parámetros de crecimiento para L. plantarum en presencia de $\Omega 3$.

Table 1. Growth parameters of probiotic strain L. plantarum in presence of $\Omega 3$.

\begin{tabular}{|c|c|c|c|}
\hline $\begin{array}{l}\text { Aceite de linaza } \\
\text { en medio de cultivo (\%) }\end{array}$ & $\begin{array}{l}\text { Fase de } \\
\text { acondicionamiento(h) }\end{array}$ & Tasa de crecimiento $\left(\mathrm{DO}_{620} / \mathrm{s}\right)$ & Fase estacionaria $\left(\mathrm{DO}_{620}\right)$ \\
\hline 0 & $2.33 \pm 0.09^{a}$ & $3.5 \mathrm{E}-05 \pm 0.1 \mathrm{E} 06^{\mathrm{a}}$ & $0.98 \pm 0.04^{\mathrm{a}^{*}}$ \\
\hline 0.5 & $2.17 \pm 0.11^{\mathrm{a}}$ & $3.0 \mathrm{E}-05 \pm 0.1 \mathrm{E} 07^{\mathrm{b}}$ & $0.95 \pm 0.02^{\mathrm{a}}$ \\
\hline 1.0 & $1.81 \pm 0.03^{b}$ & $3.0 \mathrm{E}-05 \pm 0.2 \mathrm{E} 07^{\mathrm{b}}$ & $0.95 \pm 0.04^{\mathrm{a}}$ \\
\hline 2.0 & $1.84 \pm 0.05^{b}$ & $3.0 \mathrm{E}-05 \pm 0.1 \mathrm{E} 07^{\mathrm{b}}$ & $0.95 \pm 0.03^{\mathrm{a}}$ \\
\hline 4.0 & $1.80 \pm 0.05^{b}$ & $3.1 \mathrm{E}-05 \pm 0.2{\mathrm{E} 07^{\mathrm{b}}}$ & $0.94 \pm 0.04^{\mathrm{a}}$ \\
\hline 8.0 & $1.87 \pm 0.07^{b}$ & $2.8 \mathrm{E}-05 \pm-0.3 \mathrm{E} 07^{\mathrm{b}}$ & $0.95 \pm 0.02^{\mathrm{a}}$ \\
\hline
\end{tabular}

*Distintas letras en una misma columna indican diferencia significativa $(p<0.05)$.

*Different letters indicate significant differences $(p<0.05)$ between column groups.

en el medio fue de 1 a $8 \%$, independientemente de la concentración utilizada. Estos resultados podrían ser un indicio de que la presencia de los ácidos $\Omega 3$ estaría favoreciendo el crecimiento de L. plantarum. Sin embargo, al analizar los cambios en unidades de cambio de absorbancia/unidad de tiempo, se observó una pequeña pero significativa $(p<0.05)$ disminución, en presencia del aceite de linaza, independientemente de la concentración utilizada, indicando que la velocidad de la fase de crecimiento exponencial fue $15 \%$ menor en presencia del aceite. Por otro lado, no se observaron cambios ( $p>0.05$ ) durante la fase estacionaria.Eratte et al. (2016b) observaron que $L$. casei crece mejor en presencia de aceite, independientemente de la fuente utilizada. Con los resultados obtenidos en este estudio no puede concluirse lo mismo, ya que, aunque se observó una disminución del 21 $\%$ en el tiempo requerido para que $L$. plantarum empezara a multiplicarse, su capacidad para hacerlo fue más lenta durante la fase de acondicionamiento. Por lo anterior, es necesario hacer análisis complementarios.

\section{Condiciones de encapsulamiento}

Para determinar la combinación adecuada de alginato-pectina que formara cápsulas consistentes, se hicieron 3 tipos de cápsulas; de pectina al $2 \%$ (T1); de alginato (1.0 \%)-pectina (2\%) relación 2:1 (T2) y de alginato (1.5 \%)-pectina (2\%), relación 2:1 (T3). En la Figura 2, se presentan las observaciones al estereoscopio, de T1, T2 y T3. Las cápsulas $\mathrm{T} 1$, que contenían únicamente pectina, mostraron escasa consistencia y forma variada (Figura 2A). Por otro lado, al añadir alginato (T2 y T3), la consistencia mejoró, obteniéndose mayor firmeza y forma cuasi-esférica en las cápsulas T3 (Figura 2C). Por tanto, se escogió este tratamiento como la matriz encapsulante para continuar el estudio.Una posible causa de la escasa consistencia de las cápsulas de pectina utilizada en este estudio, es la variación en la cantidad de grupos metoxilos contenidos en el ácido galacturónico ( 25 al 40 $\%)$, así como la distribución de los mismos. La interacción de las pectinas con $\mathrm{Ca}^{+2}$ se establecen con los grupos carboxilo $\left(\mathrm{COO}^{+}{ }^{+} \mathrm{Ca}^{+} \mathrm{COO}\right)$. De esta manera, entre mayor sea el número de metoxilaciones $\left(\mathrm{COOCH}_{3}\right)$ y más cercanas estén unas de

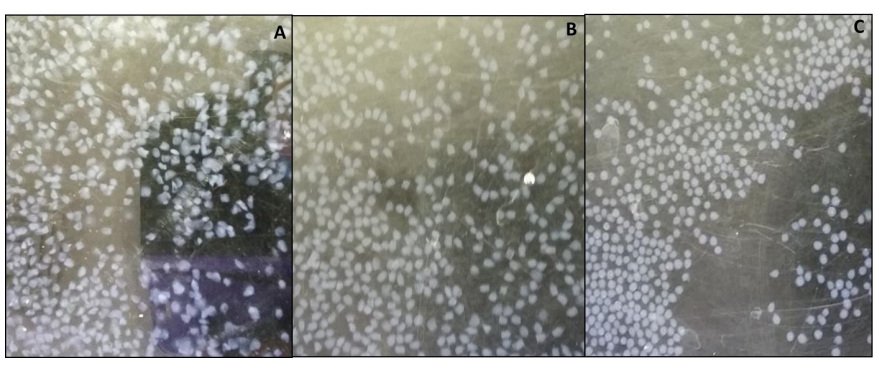

Figura 2. Imágenes representativas de las microcápsulas sintetizadas con A) pectina al $2 \%$; B) mezcla alginato (1.0\%)-pectina (2\%) relación 2:1;C) mezcla alginato (1.5\%)-pectina (2\%), relación 2:1.

Figure 2. Representative images of microcapsules synthetized with A) 2 $\%$ pectin alone; $\mathrm{B}$ ) alginate $(1.0 \%)$-pectin $(2 \%)$ mixture ratios of $2: 1 ; \mathrm{C})$ alginate (1.5\%)-pectin (2\%) mixture ratios of 2:1.

otras, la posibilidad de entrecruzamiento será menor, formándose geles más débiles y cápsulas menos consistentes. Esta posibilidad disminuye cuando se utiliza alginato, donde la interacción con $\mathrm{Ca}^{+2}$ puede llegar a ser más homogénea, debido a que se establece con regiones homopoliméricas del ácido gulurónico, donde los grupos carboxilo no están modificados con grupos metilos y, por tanto, no existe impedimento para la interacción (Guo y Kaletunç, 2016).

En la Figura 3 se presentan las diferentes combinaciones de matriz-probiótico-aceite. Al utilizar la proporción 5.0:2.5:2.5 (matriz-probiótico- $\Omega 3$ ), se obtuvieron cápsulas poco uniformes, de tamaño variado e inconsistentes (Figura 3A). La combinación 5.0:1.0:2.5 obtuvo tamaños más unifor-

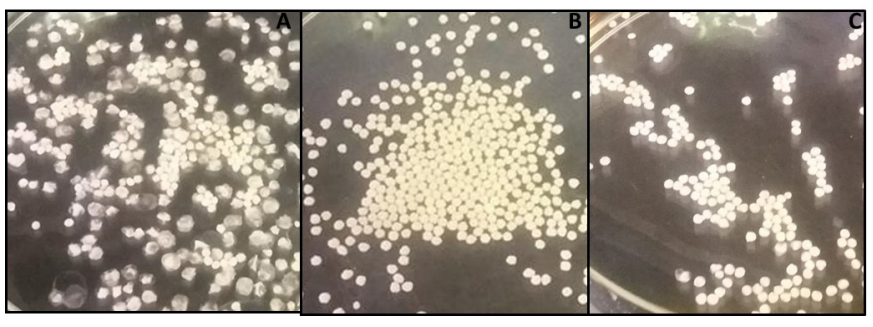

Figura 3. Imágenes representativas de las microcápsulas de alginato-pectina (matriz) cargadas con L. plantarum y $\Omega 3$. A) relación matriz:probiótico:pectina 5.0:2.5:2.5. B) 5.0:1.0: 2.5. C) 5.0: 1.0:1.0.

Figure 3. Representative images of alginate-pectin microcapsules (matrix) loaded with $L$. plantarum and $\Omega 3$. A); matrix: probiotic: $\Omega 3$ ratios 5.0:2.5:2.5. B) 5.0:1.0: 2.5. C) 5.0: 1.0:1.0. 
mes y mejor consistencia (Figura 3B). Sin embargo, después de $1 \mathrm{~h}$ se observó que el aceite migraba al exterior, por lo que finalmente se escogió la combinación 5.0:1.0:1.0 que fue similar en consistencia y distribución de tamaños, sin que se observara migración de aceite (Figura 3C). El encapsulamiento con alginato utilizando el mecanismo de gelificación externa es el más utilizado en la industria alimentaria. Implica extruir una suspensión de alginato que contiene el material a encapsular, sobre un baño de cloruro de calcio (Burgain et al., 2011). El calcio interacciona principalmente con el alginato superficial de las gotas formadas por la extrusión y en menor grado, con el interior de la gota, permitiendo la formación de una cápsula flexible (Leong et al., 2016). Sin embargo, cuando la relación de alginato disminuye, el entrecruzamiento es más débil, ocurriendo un rompimiento de las cápsulas, de su producción o durante su almacenamiento y utilización (Comunian y Favaro-Trinade, 2016). Un efecto adicional es que se forman poros más grandes en la cápsula que pueden permitir la migración del compuesto encapsulado (Leong et al., 2016).

Este comportamiento puede explicar la salida del aceite en las cápsulas elaboradas con la combinación 5.0:1.0:2.5. Una manera de mejorar la retención de aceites encapsulados es añadiendo un recubrimiento o capa adicional a la superficie de la cápsula (Mokarram et al., 2009). Otros investigadores han probado con éxito la técnica de coaservación promoviendo interacciones entre carbohidratos y proteínas (Comunian y Favaro-Trinade, 2016). Por ejemplo, Eratte et al. (2015) co-encapsularon L. casei 341 con aceite de atún utilizando una matriz de goma arábiga y proteínas de suero de leche, obteniendo coacervados estables, sin observar pérdida de aceite. El encapsulamiento de L. casei y aceite de pescado por la técnica de secado por aspersión tampoco produjo pérdidas de aceite durante el almacenamiento. Sin embargo, las altas temperaturas de secado, promovieron su oxidación (Eratte et al., 2016a).

\section{Caracterización de los microencapsulados obtenidos por el método semi automatizado}

En la Tabla 2, se observa que la presencia del aceite de linaza no afectó el tamaño, obteniéndose diámetros entre aproximadamente 150 a $200 \mu \mathrm{m}$. Tampoco la carga superficial de los microencapsulados cambio considerablemente,

Tabla 2. Caracterización de los microencapsulados de L. plantarum y $\Omega 3$ en alginato-pectina.

Table 2. Characterization of L. plantarum and omega-3 microencapsulated in alginate-pectin matrix.

\begin{tabular}{lcc}
\hline Característica & $\begin{array}{c}\text { Microcápsulas } \\
\text { con L.plantarum }\end{array}$ & $\begin{array}{c}\text { Microcápsulas con } \\
\text { L.plantarum }+\Omega 3\end{array}$ \\
\hline Diámetro $(\mu \mathrm{m})$ & $131 \pm 73.6^{\mathrm{a}}$ & $138 \pm 78.2^{\mathrm{a}}$ \\
Carga superficial $(\mathrm{mV})$ & $-4.77 \pm 0.6^{\mathrm{a}}$ & $-4.62 \pm 0.2^{\mathrm{a}}$ \\
Forma & esférica & esférica \\
\hline
\end{tabular}

*Distintas letras en una misma fila indican diferencia significativa $(p<0.05)$. *Different letters indicate significant differences $(p<0.05)$ between line groups.

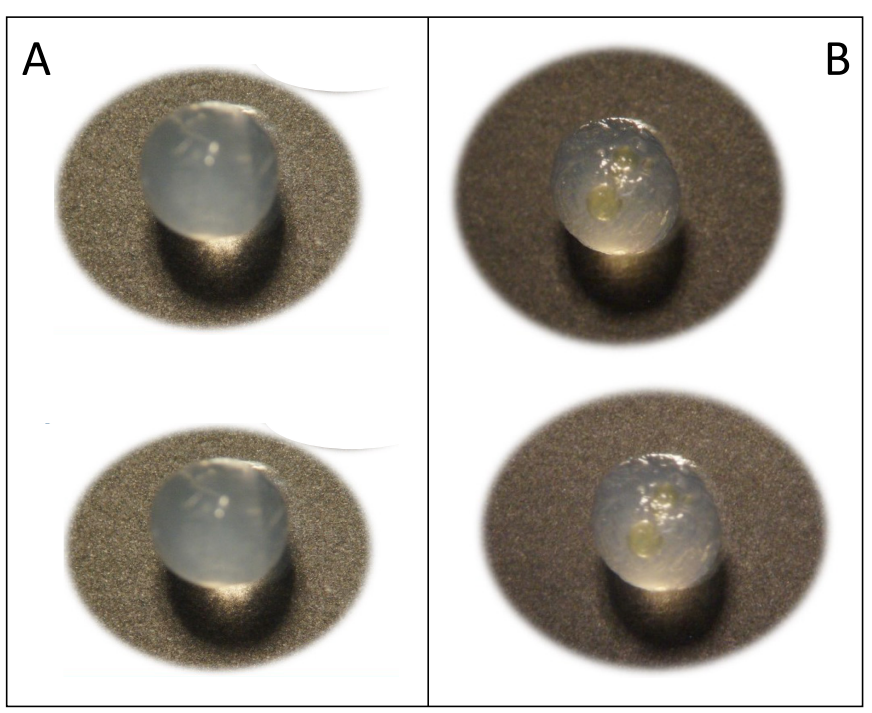

Figura 4. Imágenes representativas de las microcápsulas de alginato-pectina obtenidas por el método semi-automático. A) microcápsulas vacías. B) microcápsulas cargadas con L. plantarum y $\Omega 3$.

Figure 4. Representative images of alginate-pectin microcapsules obtained by the semi-automatic method. A) empty microcapsules. B) microcapsules loaded with $L$. plantarum and $\Omega 3$.

encontrando valores entre -4.17 y $-4.83 \mathrm{mV}$. Las micrografías mostraron que en ambos casos se obtuvieron microcápsulas esféricas (Figura 4), observándose el aceite de linaza en el centro de la cápsula (Figura 4 B). En la gelación iónica, la forma de las cápsulas está directamente relacionada con el entrecruzamiento de la matriz, cuando éste es menor, las cápsulas son menos consistentes y adquieren formas ovoides o irregulares (Leong et al., 2016), como las que se presentaron al utilizar únicamente pectina para el encapsulamiento. Otros trabajos en que se han co-encapsulado $\Omega 3$ y probióticos implican técnicas diferentes como la coaservación y el secado por aspersión donde las cápsulas resultaron amorfas (Eratte et al., 2016 a; 2015).

El tamaño de la cápsula también varía dependiendo de la técnica de encapsulamiento; en el caso de la gelación iónica semiautomatizada, se obtienen tamaños más homogéneos (Leong et al., 2016), como en este estudio. Una desventaja de las cápsulas mayores a $100 \mu \mathrm{m}$ es que pueden impartir la sensación de arenosidad, cuando se añaden a alimentos líquidos (Burgain et al., 2011). Una alternativa es deshidratar las cápsulas y añadirlas a alimentos secos, lo cual es una tendencia que cada día gana más interés en la industria de alimentos (Leong et al., 2016).

\section{Eficiencia de encapsulamiento}

Para estimar la cantidad de L. plantarum atrapado en los microencapsulados se determinó la viabilidad por cuenta en placa, antes y después del encapsulamiento. Las cuentas iniciales añadidas a los alginatos, fluctuaron entre 8.40 a 8.66 Log UFC/mL. Después de encapsular al probiótico, se observaron cuentas de 7.8 a 7.9 Log UFC/g de cápsulas (92 \%) y de 7.14 a 7.4 Log UFC/g de cápsulas (85 \%), en los microencapsulados de $L$. plantarum en ausencia y presencia de $\Omega 3$, respectivamente. Otros autores han observado eficiencias 
de encapsulamiento desde 90 hasta $99 \%$ cuando se usa el método de extrusión de alginatos, (Mokarram et al., 2009). El menor grado de atrapamiento del probiótico en este trabajo pudo deberse a una formación más lenta de las cápsulas provocada por la presencia de pectinas y por la de aceite.

\section{Efecto de la liofilización y del almacenamiento en conge- lación en la viabilidad del $L$. plantarum microencapsulado}

La Figura 5 muestra la viabilidad del L. plantarum, encapsulado y co-encapsulado con omega-3 después de liofilizar las cápsulas y después de almacenarlas durante 30 días a $-20^{\circ} \mathrm{C}$. Al liofilizar los encapsulados se observó una disminución de las cuentas de L. plantarum de 0.39-0.41 Log UFC/g de cápsula, mientras que al liofilizar los co-encapsulados conteniendo $\Omega 3$, la disminución fue de 0.6 a $0.8 \mathrm{Log} \mathrm{UFC} / \mathrm{g}$ de cápsula.Callewaert et al. (2007) compararon el efecto de la liofilización en la superficie de microcápsulas sintetizadas con diferentes matrices. Encontraron que las más sensibles a este proceso, fueron las obtenidas con alginato, debido a que las esferas se encogían y algunas presentaban roturas. Otros investigadores encontraron que al recubrirlas cápsulas de alginato la viabilidad de $L$. paracasei, después de liofilizar las cápsulas que lo contenían, disminuyó en el orden de 0.3 Log UFC/mL. En este trabajo no se recubrieron las cápsulas; sin embargo, la presencia de pectina pudiera estar protegiendo

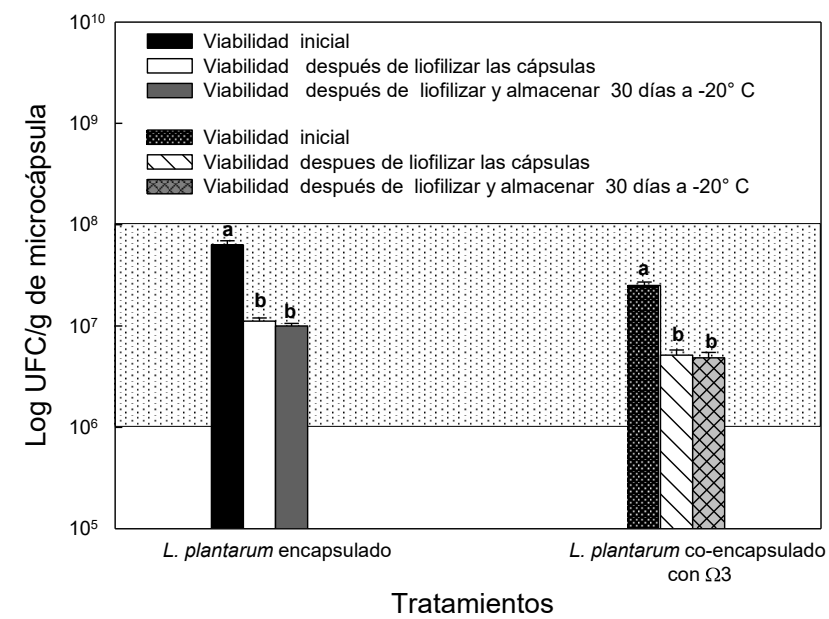

Figura 5. Efecto en la viabilidad de L. plantarum debido al co-encapsulamiento con $\Omega 3$, después de liofilizar las cápsulas y después de 30 días de almacenamiento a $-20^{\circ} \mathrm{C}$. El recuadro marcado indica las cuentas de probióticos recomendadas para ejercer un efecto benéfico a la salud. Diferentes letras muestran diferencia significativa, dentro de un mismo tratamiento. Figure 5. Effect of co-encapsulation with $\Omega 3$, lyophilization of capsules and after 30 days of storage at $-20^{\circ} \mathrm{C}$ on the viability of $L$. plantarum. The marked box indicates the probiotic accounts recommended to have a beneficial effect on health. Different letters show significant difference, within the same treatment.

la superficie de los microencapsulados. No obstante, se requieren nuevas investigaciones donde se contraste el efecto de la viabilidad con cápsulas que contengan únicamente alginato y se desarrolle un estudio de los cambios superficiales en ambos tipos (alginato, alginato-pectina) de matrices. La presencia del aceite de linaza afectó la viabilidad del $L$. plantarum al liofilizar los microencapsulados. Este efecto también fue observado por Silva et al. (2016), en presencia de aceite de girasol. Sin embargo, como se observa en la Figura 5, a pesar de la disminución observada, las cuentas de L. plantarum se mantuvieron dentro de las indicadas para producir un efecto benéfico a la salud (Burgain et al., 2011). En la Figura 5 también se observa que después de 30 días de almacenamiento a $-20^{\circ} \mathrm{C}$ la viabilidad del probiótico no se afectó, indicando que la matriz es estable a este efecto. Sin embargo, debe mencionarse que, cuando se almacenan microencapsulados a estas condiciones, es muy importante protegerlos de los cambios de humedad y actividad de agua, para evitar cambios en la disponibilidad de agua que produzcan reacciones que puedan afectar al probiótico (Burgain et al., 2011).

\section{Estabilidad del aceite encapsulado}

La Figura 6 compara el índice de acidez del aceite encapsulado con el del libre, durante 20 días de almacenamiento. En ambos casos se observaron índices bajos indicando que la rancidez producida por el ataque de lipasas fue menor. Sin embargo, este tipo de rancidez fue menor $(p<$ 0.05 ) en el aceite encapsulado, que, en el libre, lo cual es un indicio de que la matriz podría estar protegiendo al aceite.

El tipo de rancidez más frecuente en los ácidos grasos insaturados es la rancidez oxidativa que puede estimarse determinando el índice de peróxidos. Los valores iniciales de peróxidos fueron de $0.4 \pm 0.01$ meq $\mathrm{O}_{2} / \mathrm{kg}$, tanto en el aceite libre como en el encapsulado y lo mismo sucedió durante los días restantes de almacenamiento. Se ha observado que el aceite de linaza es susceptible a la rancidez oxidativa y que esta puede seguir ocurriendo, aunque más lentamente, a temperaturas de congelación, como los $-20^{\circ} \mathrm{C}$ a los que se almacenaron las cápsulas y el aceite. El hecho de que no pudieran determinarse los peróxidos durante la mayor parte del almacenamiento podría ser un indicio de estabilidad o de que la técnica utilizada no fue suficientemente sensible, por lo que es necesario realizar pruebas de rancidez acelerada

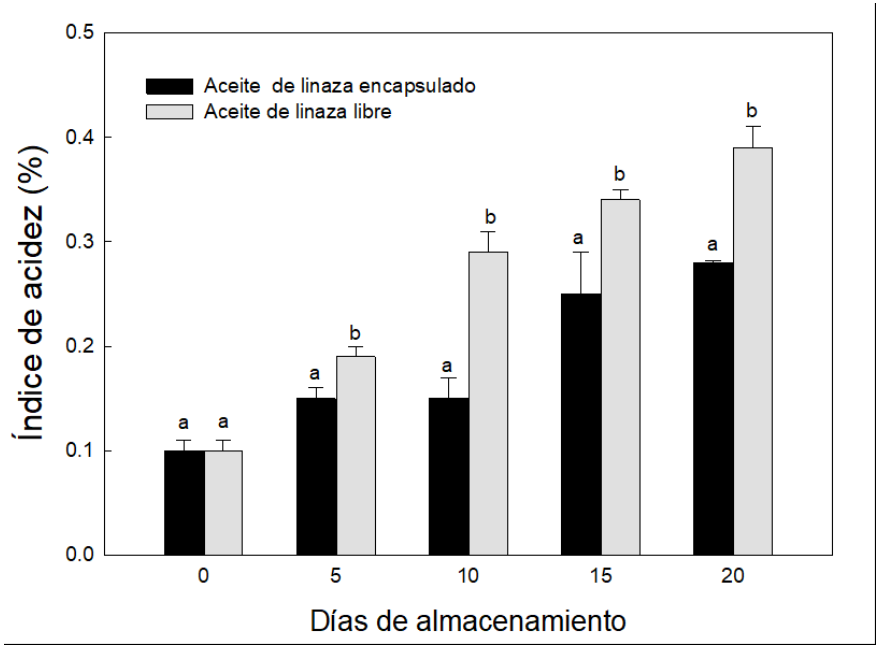

Figura 6. Efecto del encapsulamiento en el índice de acidez de los $\Omega 3$. Figure 6. Effect of encapsulation over acidity index of $\Omega 3$. 
para poder determinar si en realidad el encapsulamiento con alginato-pectina de bajo metoxilo puede proteger al aceite de linaza de este tipo de deterioro.

\section{CONCLUSIÓN}

Los resultados obtenidos en el presente trabajo indican que es posible co-encapsular probióticos y $\Omega 3$ por gelación iónica utilizando una relación $2: 1$ de alginato (1.5 $\%)$ y pectina (2\%) siempre y cuando se encuentre la relación adecuada matriz: probiótico: aceite. Utilizando la relación 5.0:1.0:1.0, se obtuvieron cápsulas firmes, estables y que retuvieron el aceite después de almacenarlas durante 30 días a $-20{ }^{\circ} \mathrm{C}$, sin afectar la sobrevivencia del probiótico ni disminuir la estabilidad a la oxidación de los $\Omega 3$. Lo anterior es un indicio de que este tipo de co-encapsulados tienen potencial para utilizarse en el desarrollo de alimentos funcionales.

Por otro lado, no se encontró evidencia determinante de que los $\Omega 3$ estimulen el crecimiento de $L$. plantarum libres (sin encapsular), ni al co-encapsularlos y almacenarlos durante 30 días. Sin embargo, se observó una ligera disminución de la sobrevivencia del probiótico en presencia de los $\Omega 3$, durante la liofilización de las cápsulas. A pesar de lo anterior, las cuentas microbianas se mantuvieron dentro de los rangos establecidos para proporcionar un efecto benéfico a la salud del consumidor debido a un posible efecto protector de la pectina. Lo anterior es un indicio de que la combinación alginato-pectina puede resultar efectiva en el co-encapsulamiento de bioactivos y probióticos. Sin embargo, se requieren estudios complementarios para probarlo.

\section{AGRADECIMIENTOS}

Se agradece al Consejo Nacional de Ciencia y Tecnología (CONACYT) por el financiamiento al proyecto CB-2011169658, así como por la beca otorgada a la estudiante de licenciatura. Los autores agradecen a la Plataforma Analítica Institucional de CIAD, por las facilidades prestadas para la realización del estudio.

\section{REFERENCIAS}

Avramenko, N. A., Chang, C., Low, N. H., y Nickerson, M. T. (2016). Encapsulation of flaxseed oil within native and modified lentil protein-based microcapsules. Food Research International, 81, 17-24.

Baker, E. J., Miles, E. A., Burdge, G. C., Yaqoob, P., y Calder, P. C. (2016). Metabolism and functional effects of plant-derived omega-3 fatty acids in humans. Progress in Lipid Research, 64, 30-56.

Barreto, F. M., ColadoSimão, A. N., Morimoto, H. K., Batisti Lozovoy, M. A., Dichi, I., y Helena da Silva Miglioranza, L. (2014). Beneficial effects of Lactobacillus plantarum on glycemia and homocysteine levels in postmenopausal women with metabolic syndrome. Nutrition, 30(7-8), 939-942.Barros, R., Moreira, A., Fonseca, J., Delgado, L., Graça Castel-Branco, M., Haahtela, T., Lopes, C., y Moreira, P. (2011). Dietary intake of a-linolenic acid and low ratio of n-6:n-3 PUFA are associated with decreased exhaled $\mathrm{NO}$ and improved asthma control.
British Journal of Nutrition, 106(3), 441-450.

Ben Salah, R., Trabelsi, I., Hamden, K., Chouayekh, H., y Bejar, S. (2013). Lactobacillus plantarum TN8 exhibits protective effects on lipid, hepatic and renal profiles in obese rat. Anaerobe, 23, 55-61.

Burgain, J., Gaiani, C., Linder, M., y Scher, J. (2011). Encapsulation of probiotic living cells: From laboratory scale to industrial applications. Journal of Food Engineering, 104(4), 467-483.

Callewaert, M., Laurent-Maquin, D., y Edwards-Lévy, F. (2007). Albumin-alginate-coated microspheres: resistance to steam sterilization and to lyophilization. International journal of pharmaceutics, 344(1-2), 161-164.

Carneiro, H. C. F., Tonon, R. V., Grosso, C. R. F., y Hubinger, M. D. (2013). Encapsulation efficiency and oxidative stability of flaxseed oil microencapsulated by spray drying using different combinations of wall materials. Journal of Food Engineering, 115(4), 443-451.

Choi, E. A., y Chang, H. C. (2015). Cholesterol-lowering effects of a putative probiotic strain Lactobacillus plantarum EM isolated from kimchi. LWT - Food Science and Technology, 62(1, Part 1), 210-217.

Chowdhury, R., Warnakula, S., Kunutsor, S., Crowe, F., Ward, H. A., Johnson, L., y Di Angel Antonio, E. (2014). Association of dietary, circulating, and supplement fatty acids with coronary risk: A systematic review and meta-analysis. Annals of Internal Medicine, 160(6), 398-406.Comunian, T. A., y Favaro-Trindade, C. S. (2016). Microencapsulation using biopolymers as an alternative to produce food enhanced with phytosterols and omega-3 fatty acids: A review. Food Hydrocolloids, 61, 442-457.

Eratte, D., Gengenbach, T. R., Dowling, K., Barrow, C. J., y Adhikari, B. (2016a). Survival, oxidative stability, and surface characteristics of spray dried co-microcapsules containing omega-3 fatty acids and probiotic bacteria. Drying Technology, 34(16), 1926-1935.Eratte, D., McKnight, S., Gengenbach, T. R., Dowling, K., Barrow, C. J., y Adhikari, B. P. (2015). Co-encapsulation and characterization of omega-3 fatty acids and probiotic bacteria in whey protein isolate-gum Arabic complex coacervates. Journal of Functional Foods, 19, Part B, 882-892.Eratte, D., Wang, B., Dowling, K., Barrow, C. J., y Adhikari, B. (2016b). Survival and fermentation activity of probiotic bacteria and oxidative stability of omega-3 oil in co-microcapsules during storage. Journal of Functional Foods, 23, 485-496.

FAO/WHO, 2002. Joint Working Group Report on Drafting Guidelines for the Evaluation of Probiotic in Food. Canada, London, Ontario. April 30 and May 1.

Ghidurus, M., Calin, A., Beciu, S., y Ladaru, R. (2015). Functional foods, probiotics and their role on human health. Journal of Biotechnology, 208, Supplement, S67. Ghidurus, M., Calin, A., Beciu, S., y Ladaru, R. (2015). Functional foods, probiotics and their role on human health. Journal of Biotechnology, 208, Supplement, S67.

Guo, J., y Kaletunç, G. (2016). Dissolution kinetics of pH responsive alginate-pectin hydrogel particles. Food Research International, 88, Part A, 129-139.

Hajlaoui, H., Mighri, H., Aouni, M., Gharsallah, N., y Kadri, A. (2016). Chemical composition and in vitro evaluation of antioxidant, antimicrobial, cytotoxicity and anti-acetylcholinesterase properties of Tunisian Origanum majorana L. essential oil. Microbial Pathogenesis, 95, 86-94. 
Ilavenil, S., Kim, H. D., Valan Arasu, M., Srigopalram, S., Sivanesan, R., y Choi, C. K. (2015). Phenyllactic acid from Lactobacillus plantarum promotes adipogenic activity in 3T3-L1 adipocyte via up-regulation of PPAR- $\gamma 2$. Molecules, 20(8).

Jeun, J., Kim, S., Cho, S.-Y., Jun, H.-j., Park, H.-J., Seo, J.-G., y Lee, S.-J. (2010). Hypocholesterolemic effects of Lactobacillus plantarum KCTC3928 by increased bile acid excretion in C57BL/6 mice. Nutrition, 26(3), 321-330.

Jiménez P, P., Masson S, L., y Quitral R, V. (2013). Composición química de semillas de chía, linaza y rosa mosqueta y su aporte en ácidos grasos omega-3. Revista chilena de nutrición, 40, 155-160.

Leong, J.-Y., Lam, W.-H., Ho, K.-W., Voo, W.-P., Lee, M. F.-X., Lim, H.-P., y Chan, E.-S. (2016). Advances in fabricating spherical alginate hydrogels with controlled particle designs by ionotropic gelation as encapsulation systems. Particuology, 24, 44-60.

Li, H., Thuy Ho, V.T., Turner, M. S., y Dhital, S. (2016). Encapsulation of Lactobacillus plantarum in porous maize starch. LWT-Food Science and Technology, 74, 542-549.

Mokarram, R. R., Mortazavi, S. A., Najafi, M. B. H., y Shahidi, F. (2009). The influence of multi stage alginate coating on survivability of potential probiotic bacteria in simulated gastric and intestinal juice. Food Research International, 42(8), 1040-1045.

Morales, M. E., y Ruiz, M. A. (2016). 16 - Microencapsulation of probiotic cells: applications in nutraceutic and food industry A2 - Grumezescu, Alexandru Mihai Nutraceuticals (pp. 627668): Academic Press.

Rafiee, M., Sotoudeh, G., Djalali, M., Alvandi, E., Eshraghian, M., Sojoudi, F., y Koohdani, F. (2016). Dietary $\omega$-3 polyunsaturated fatty acid intake modulates impact of Insertion/Deletion polymorphism of $A$ poB gene on obesity risk in type 2 diabetic patients. Nutrition, 32(10), 1110-1115.

Rajam, R., y Anandharamakrishnan, C. (2015). Spray freeze drying method for microencapsulation of Lactobacillus plantarum. Journal of Food Engineering, 166, 95-103.

Sampson, S. (2015). Functional Foods: The Connection Between Nutrition, Health, and Food Science. Journal of Nutrition Education and Behavior, 47(1), 117-119.

Silva, M. P., Tulini, F. L., Ribas, M. M., Penning, M., Favaro-Trindade, C. S., y Poncelet, D. (2016). Microcapsules loaded with the probiotic Lactobacillus paracasei BGP-1 produced by coextrusion technology using alginate/shellac as wall material: Characterization and evaluation of drying processes. Food Research International, 89(1), 582-590.

Slover, H. T., y Lanza, E. (1979). Quantitative analysis of food fatty acids by capillary gas chromatography. Journal of the American Oil Chemists Society, 56(12), 933-943.

Stevenson, C., Blaauw, R., Fredericks, E., Visser, J., y Roux, S. (2014). PP137-SUN: Randomized Clinical Trial: Effect of Lactobacillus plantarum 299V on Symptoms of Irritable Bowel Syndrome. Clinical Nutrition, 33, S71.

Woo, J.-Y., Gu, W., Kim, K.-A., Jang, S.-E., Han, M. J., y Kim, D.-H. (2014). Lactobacillus pentosus var. plantarum C29 ameliorates memory impairment and inflammation in a d-galactoseinduced accelerated aging mouse model. Anaerobe, 27, 22-26.

Wu, Y., Zhu, C., Chen, Z., Chen, Z., Zhang, W., Ma, X., Wang, L., Yang, X., y Jiang, Z. (2016). Protective effects of Lactobacillus plantarum on epithelial barrier disruption caused by enterotoxigenicEscherichiacoli in intestinal porcineepithelial cells. Veterinary Immunology and Immunopathology, 172, 5563. 